UCRL-ID-121668

\title{
Region-of-Interest Cone-Beam Computed Tomography
}

S. G. Azevedo

P. Rizo

P. Grangeat
RECEIVED

NOV 171995

OSAI

June 1995

This is an informal report intended primarily for internal or limited external distribution. The opinions and conclusions stated are those of the author and may or may not be those of the Laboratory.

Work performed under the auspices of the U.S. Department of Energy by the Lawrence Livermore National Laboratory under Contract W-7405-Eng-48. 


\section{DISCLAIMER}

This document was prepared as an account of work sponsored by an agency of the United States Government. Neither the United States Government nor the University of California nor any of their employees, makes any warranty, express or implied, or assumes any legal liability or responsibility for the accuracy, completeness, or usefulness of any information, apparatus, product, or process disclosed, or represents that its use would not infringe privately owned rights. Reference herein to any specific commercial product, process, or service by trade name, trademark, manufacturer, or otherwise, does not necessarily constitute or imply its endorsement, recommendation, or favoring by the United States Government or the University of California. The views and opinions of authors expressed herein do not necessarily state or reflect those of the United States Government or the University of California, and shall not be used for advertising or product endorsement purposes.

This report has been reproduced directly from the best available copy.

Available to DOE and DOE contractors from the Office of Scientific and Technical Information

P.O. Box 62, Oak Ridge, TN 37831

Prices available from (615) 576-8401, FTS 626-8401

Available to the public from the National Technical Information Service

U.S. Department of Commerce 5285 Port Royal Rd., Springfield, VA 22161 


\section{DISCLAIMER}

Portions of this document may be illegible electronic image products. Images are produced from the best available original document. 


\title{
REGION-OF-INTEREST CONE-BEAM COMPUTED TOMOGRAPHY
}

\author{
Stephen AZEVEDO', Philippe RIZO ${ }^{2}$, Pierre GRANGEAT ${ }^{2}$ \\ ${ }^{1}$ Lawrence Livermore National Laboratory \\ P. O. Box 808 L-495, Livermore, California, 94551 - USA \\ azevedo3@llnl.gov \\ ${ }^{2}$ LETI (CEA-Technologies Avancées) \\ DSYS - CEN/G - 17, avenue des Martyrs \\ 38054 Grenoble Cedex 9 - France
}

\begin{abstract}
A methodology for solving the general cone-beam regionof-interest (ROI) problem on a circular trajectory is presented using the mathematical framework described by Grangeat [1]. The algorithm, called Radon-ROI, takes scans at two different resolutions-low resolution covering the entire object and high resolution covering only the ROI-and combines the scans in both projection and Radon spaces so that the ROI is reconstructed at high resolution without artifacts from missing-data, undersampling, or cone-beam errors. A circular source trajectory is assumed and the object must have low spatial frequencies outside the ROI. Simulated and experimental results of the Radon-ROI code show marked improvement on resolution within the ROI.
\end{abstract}

\section{INTRODUCTION}

The region of interest problem in computed tomography (CT), where only a small region of the patient is irradiated and imaged, has been extensively studied for the two-dimensional case. Although a complete set of projections is theoretically required for $2 \mathrm{D} C \mathrm{CT}$ reconstruction, the ROI problem has been solved in several ways. Some of the best ROI reconstruction method$s$ involve the use of models or extra measurements for regeneration of the missing ray-sums. One can use a model of the object to complete the missing data $[2,3]$, or use assumed models to estimate a best fit to the missing data $[4,5]$. The difficulty of these methods is in the

THIS RESEARCH WAS SUPPORTED IN PART BY THE COMMISSION OF THE EUROPEAN COMMUNITIES BRITEEURAM PROJECT CONTRACT GR-771.528 C.E.E. AND UNDER THE AUSPICES OF THE U. S. DEPARTMENT OF ENERGY UNDER DOE CONTRACT W-7405-ENG-48. (LLNL). choice and alignment of the model to the data; errors in these steps cause reconstruction errors and no way of quantifying the artifacts. A more heuristic method is to acquire a second scan that completely covers the object, but at low resolution (LR), and to combine this data with the high resolution (HR) ROI projections or reconstructions [6] using interpolation. When a complete set of ray-sums is estimated, by whatever method, reconstruction of the ROI image is straight-forward using filtered backprojection or other known $2 \mathrm{D}$ technique.

However, extending the above methods to the 3D cone-beam ROI problem is not straight-forward. The model-based methods could be extended with the use of 3D models to describe the object, but now the modeling and alignment problems are even more difficult. The heuristic method of combining two scans with differing resolution is not directly possible, in general, since the LR scan will not contain the missing ray-sums needed by the HR scan. Only those ray-sums in the mid-plane (the plane containing the source trajectory) can be interpolated directly from the LR scan. Normally, no ray-sums off of the mid-plane are colinear between the two scans, and hence cannot be combined in the same sense.

Still, the main advantage of cone-beam 3D CT over parallel-beam lies in the ability to achieve higher resolution of the object through geometric magnification. At relatively low cone-angles (less than \pm 12 degrees) [7], algorithms exist that perform reasonably well for circular source trajectories in spite of the so-called "shadow zone" of missing data.

In this paper, we develop a new algorithm for the conebeam ROI algorithm and test it in several cases. We will first describe the method, then follow with some results. 


\section{GENERAL APPROACH}

Extending the 2D heuristic approach above to 3D is one possible approach for cone-beam ROI CT we will call reconstruction/reprojection. The LR scan could be reconstructed for a full but crude view of the object, then mathematically reprojected at small sample spacing to generate missing data from the ROI scan. This can be time-consuming and still not address other missing data in the high-resolution scan (i.e., the shadow zones above and below the source trajectory plane in Radon space).

We propose a similar method, but using the methodology described by Grangeat [1] for cone-beam reconstruction. In this way, we have an intermediate space-the 3D Radon domain or $\mathcal{D} \mathcal{R}$-space-that can be used for combining LR and HR data. We will show how the reprojected ray-sums can be found more rapidly through an intermediate space (Radon space), and how performing a second combination process in this intermediate space will reduce cone-beam artifacts. Therefore, two new features of this technique, increased speed and reduced artifacts, make it more desirable than the simple reconstruction/reprojection method.

\section{CONE-BEAM RECONSTRUCTION}

The reconstruction process proposed by Grangeat [1], called the Radon algorithm, starts with the $x$-ray transform of the 3D volume $f()$, which is the mathematical representation of the cone-beam data acquisition process:

$$
\mathcal{X} f(S, A)=\int_{0}^{\infty} f\left(S+a \frac{\overrightarrow{S A}}{\|\overrightarrow{S A}\|}\right) d a
$$

for all points $A$ on the detection plane and all positions $S$ on the source trajectory.

The first step in reconstruction involves computing the 3D Radon transform of the object function defined as follows:

$$
\mathcal{R} f(\rho, \overrightarrow{\mathbf{n}})=\int_{(\overrightarrow{\mathrm{OM}} \cdot \mathbf{\mathbf { n }})=\rho} f(M) d M,
$$

where $\overrightarrow{\mathbf{n}}$ is a $3 \mathrm{D}$ unitary direction vector from the object origin, given in standard spherical coordinates $(\rho, \theta, \phi)$. The principle is to compute, and then to invert, the first derivative of the Radon transform. This uses the Radon domain as a rebinning space. The 3D Radon transform, therefore, is formed by integrating the object function over planes, rather than lines. Another way to write the Radon transform is:

$$
\mathcal{R} f(\rho, \theta, \phi)=\iiint_{-\infty}^{\infty} f(x, y, z) \delta\left[\overrightarrow{\mathbf{n}}^{T} \overrightarrow{\mathbf{x}}-\rho\right] d x d y d z .
$$

where $\overrightarrow{\mathrm{x}}$ is the vector representing a point $M(x, y, z)$ and $\delta()$ is the Dirac delta function.

Grangeat found the following exact relationship for computing the 3D Radon transform from the $x$-ray transform:

$$
\frac{\|\overrightarrow{O S}\|^{2}}{\|\overrightarrow{O S} \times \overrightarrow{\mathbf{n}}\|^{2}} \frac{\partial(\mathcal{S Y f})}{\partial \rho^{\prime}}(S, \overrightarrow{\mathbf{n}})=\frac{\partial(\mathcal{R} f)}{\partial \rho}(\overrightarrow{O S} \cdot \overrightarrow{\mathbf{n}}, \overrightarrow{\mathbf{n}})
$$

where $\rho=\|\overrightarrow{O C}\|, \rho^{\prime}=\left\|\overrightarrow{O C^{\prime}}\right\|$, and

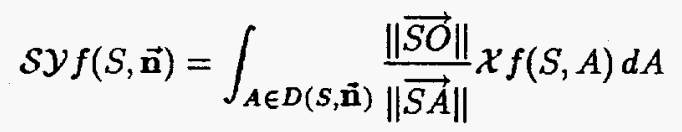

where $D(S, \overrightarrow{\mathbf{n}})$ is the intersection line between the detection plane and the plane passing through $S$ and perpendicular to $\overrightarrow{\mathbf{n}}$. Equation 4 gives an exact method to compute some values of the first derivative of the Radon transform from the x-ray transform. For notational purposes, define $\mathcal{D}$ as the partial derivative operator with respect to the first variable, so that

$$
\mathcal{D} \mathcal{R} f=\partial \mathcal{R} f / \partial \rho
$$

In the rest of this paper, references to the Radon space actually denote the first derivative of the Radon transform in $\rho$, or $\mathcal{D} \mathcal{R}$-space.

An inversion of the 3D Radon transform, then, can be given by the following equation:

$$
f(M)=-\frac{1}{4 \pi^{2}} \int_{0}^{\pi} \int_{0}^{\pi} \frac{\partial^{2} \mathcal{R} f}{\partial \rho^{2}}\left[\overrightarrow{\mathbf{n}}^{T} \overrightarrow{\mathbf{x}}, \theta, \phi\right] \sin \theta d \theta d \phi .
$$

The inverse Radon transform can be easily and efficiently implemented with existing 2D filtered backprojection algorithms.

The Radon algorithm has been shown to be accurate, efficient, and has some properties that are useful in the ROI technique.

\section{CONE-BEAM GENERATION}

Assume that a complete Radon-space representation of an object is available. We now generate computed conebeam projections from any new source position, $S$, given $\mathcal{D R} f(\rho, \theta, \phi)$. First, define "parallel lines in $z$ " as all the lines that lie in a fixed- $z$ plane. Each parallel line in $z$ can be parameterized by the polar coordinates $r$ and $\phi$ in that plane. Then define a special case of the $x$ ray transform that only represents integrals along these parallel lines in $z$ :

$$
\tilde{\mathcal{X}}_{z} f(r, \phi)=\int_{-\infty}^{\infty} f(x, y, z) \delta(x \cos \phi+y \sin \phi-r) d x d y
$$


This is called the parallel $x$-ray transform at a constant value of $z$. It is the same as if ray-sum measurements were acquired with the $\mathrm{x}$-ray source at infinity so that the incoming radiation is along parallel lines.

It can be shown that $\widetilde{\mathcal{X}}_{z} f$ is also an intermediate form of the 3D inverse Radon transform of Equation 7:

$$
\tilde{\mathcal{X}}_{z} f(r, \phi)=\frac{1}{2 \pi} \int_{0}^{\pi} \mathcal{H} \mathcal{D} \mathcal{R} f(z \cos \theta+r \sin \theta, \theta, \phi) d \theta
$$

where $\mathcal{H}$ is the Hilbert transform. This is a simple filtered backprojection and it can be used to compute any parallel ray-sum. Physically, in the Radon domain, the backprojection integration is along a circle in the $\phi$-plane (or meridian plane) defined by

$$
\rho=z \cos \theta+r \sin \theta \text {. }
$$

This circle passes through the origin and the point $(r, z, \phi)$ in cylindrical coordinates.

Using this result, any ray-sum in the volume can be computed by rotating the coordinates of 3D space and performing the same backprojection. Instead of backprojection integration along a circle aligned in Radon space, we can choose any circle passing through the origin. For example, with a different source trajectory (changed magnification), we pick the circles to correspond to the new rays in the acquisition coordinate system.

The above algorithm has been implemented and tested. It accepts cone-beam projections, computes their Radon transform, $\mathcal{D} \mathcal{R} f$, and then generates new projections at any source distance. The Hilbert transform is computed in the Fourier domain and trilinear interpolation of the Radon space is used during backprojection. In comparison with the reconstruction/reprojection method, this technique is less compute intensive because only $2 \mathrm{D}$ projections and backprojections are needed, rather than $3 \mathrm{D}$ projections and backprojections.

\section{RADON-SPACE COMBINATION}

Now the measured HR data containing only the ROI can be combined with the computed HR data using the above technique; the measured data is simply placed in the correct position of each $2 \mathrm{D}$ projection. The result of this operation is called the combined HR data. The newly combined HR projections could be directly reconstructed, as in the reprojection method, and the final reconstruction gives a better view of the ROI. However, there is another step of Radon-ROI that gives further improvement in final ROI reconstruction.

By the nature of the different cone angles involved, the shadow zone of the LR scan is smaller than that of the
HR scan. The common method for filling the missing data of the shadow zone is interpolation from its edges. However for the combined HR data this zone may be very large, so direct reconstruction of the HR torus will give large cone-beam artifacts. Instead, in Radon-ROI we fill the combined $\mathrm{HR}$ shadow zone with interpolated data from the smaller yet sparsely sampled LR shadow zone. Mixture of the LR and HR Radon spaces should diminish the cone-beam artifacts that exist in the final reconstruction while still maintaining the high spatial resolution in the ROI.

For Radon-ROI, the mixture is carried out by simply using the HR data in the high-resolution region of Radon space, and the LR data elsewhere. This includes the shadow zone of the volume where nearest neighbor interpolation of the LR torus is used. Notice that if the LR data is from a parallel projections there will be no shadow zone. In the current version of the program, the LR Radon space is bilinearly interpolated in each $\phi$-plane to match the sampling of the HR Radon space.

\section{EXPERIMENTAL RESULTS}

We have tested the Radon-ROI algorithm on several simulated objects and found it to perform well. A simulated sphere with five spherical voids is shown in vertical cross-section at the right of Figure 1. The top images (left and right) show the LR projection and Radon reconstruction respectively, while the bottom images show HR data. Notice that the HR projection requires a much large "detector" region. The middle row shows (at left) projections used in Radon-ROI; both have the same size detector, but the HR scan only shows the ROI. The Radon-ROI reconstruction at middle right shows how high resolution was achieved in the region of interest.

Using the experimental test-bed EVA industrial scanner at LETI [9], we acquired data from a cylindrical ceramic precombustion chamber of about $30 \mathrm{~mm}$ diameter and $20 \mathrm{~mm}$ height. It has a number of small cracks both at and below the surface. EVA is particularly wellsuited to this work because the source-detector distance is adjustable up to two meters and the object centerof-rotation can be placed anywhere in that range. EVA uses an IRT HOMX $161 \mathrm{x}$-ray tube source with a $10 \mu \mathrm{m}$ focal spot size and a tri-field Thompson image-intensifier detector system $(3 \mathrm{lp} / \mathrm{mm})$ with a CCD camera $\left(512^{2}\right)$ connected to the imaging computer. The detector system has a fixed diameter of about $200 \mathrm{~mm}$, so the coneangle (and, hence, the magnification) are defined by the source and object locations.

Figure 2 shows LR, Radon-ROI, and HR results from top to bottom. Despite of a small shift from LR to HR scans, presumably due to veiling glare in the im- 
age intensifier, clear increase in resolution is visible in the Radon-ROI over the LR scan. Since the projections change with the changes of magnification, various noise sources like these can cause differences in the relative ray-sum levels of the two experimental data sets. To correct for this, we used a global multiplicative mean normalization applied over each projection. Any further minor differences in local attenuation level can be minimized by local smoothing filter near the edge points, though this was not used in our experiments.

\section{SUMMARY}

The algorithm presented here, Radon-ROI, gives the best possible results for the ROI cone-beam CT problem without being excessively time-consuming. Radon-ROI is potentially superior to the reconstruction/reprojection method in terms of both speed and accuracy. It contains two novel features. First is the ability to calculate from $\mathcal{D} \mathcal{R} f$, without complete reconstruction, any raysum through an object. It is done much quicker than the reprojection method. This may be a useful component for other applications as well (e.g., cone-beam SPECT). The second feature is the ability to combine the Radon-spaces of low- and high-resolution scans to reduce the shadow-zone artifacts. Incorporation of these two features into a Radon-ROI algorithm demonstrates dramatic improvements in the reconstructed images over previous methods.

In addition, this technique can be generalized to other limited-data situations including laminography, missing angles, general truncated projections, or other source trajectories and scan geometries. One could imagine applying this idea to SPECT imaging using dual collimators that generate both parallel and cone-beam emission CT data [10].

Radon-ROI is shown to be faster than previously suggested ROI methods. It has been implemented on a Cray and tested relative to a reconstruction/reprojection method. A side benefit of the technique is a fast method of generating cone-beam projections at any orientation and any cone angle from a set of cone-beam CT measurements.

\section{REFERENCES}

[1] P. Grangeat. Analyse d'un système d'imagerie $3 D$ par reconstruction à partir de radiographies $X$ en géométrie conique. $\mathrm{PhD}$ thesis, l'Ecole $\mathrm{Na}$ tionale Superieure des Telecommunications, Grenoble, France, 1987.

[2] S. G. Azevedo, D. J. Schneberk, H. E. Martz, and M. F. Skeate. Model-based algorithms for limited- data tomography. In American Society for Nondestructive Testing (ASNT) Topical Conference on Industrial Computerized Tomography, Seattle, WA, pages 102-107, Columbus, OH, 1989. ASNT.

[3] J. W. Eberhard K. C. Tam and K. W. MitchelI. Incomplete-Data CT Image Reconstructions in Industrial Applications. IEEE Trans. Nucl. Sci., 37(3):1490-1499, June 1990.

[4] R. M. Lewitt and R. H. T. Bates. Image reconstruction from projections: III: Projection completion methods (theory). Optik, 50(2):189-204, 1978.

[5] K. Ogawa, M. Nakajima, and S. Yuta. A Reconstruction Algorithm from Truncated Projections. IEEE Trans. Medical Imaging, MI-3(1):34-40, March 1984.

[6] O. Nalcioglu, Z. H. Cho, and R. Y. Lou. Limited field of view reconstruction in computerized tomography. IEEE Trans. Nuclear Science, NS26(1):546-551, 1979.

[7] Ph. Rizo, P. Grangeat, P. Sire, P. LeMasson, and $P$. Melennec. Comparison of two three-dimensional x-ray cone-beam-reconstruction algorithms with circular source trajectories. J. Opt. Soc. Am. A, 8(10):1639-1648, October 1991.

[8] G. L. Zeng and G. T. Gullberg. A study of reconstruction artifacts in cone beam tomography using filtered backprojection and iterative EM algorithms. IEEE Trans. Nuclear Science, NS-37(2):759-767, April 1990.

[9] P. Sire, Ph. Rizo, M. Martin, and P. Grangeat. Development of an advanced 3D cone beam tomographic system. In D. O. Thompson and D. E. Chimenti, editors, Review of Progress in Quantitative Nondestructive Evaluation (NDE) 1991, volume 11, page (to be published), New York, 1992. Plenum Press. Presented at QNDE Meeting, July 28 - Aug. 2, 1991, Brunswick, ME, USA.

[10] J. Li, R. J. Jaszczak, H. Wang, K. L. Greer, and R. Coleman. Evaluation of combined cone beam with parallel hole collimation SPECT data. In $A b$ stract Book, Proceedings of the 38th Annual Meeting of the Society of Nuclear Medicine, volume 32, no. 5, page 956. Journal of Nuclear Medicine, 1991. Paper no. 201. 


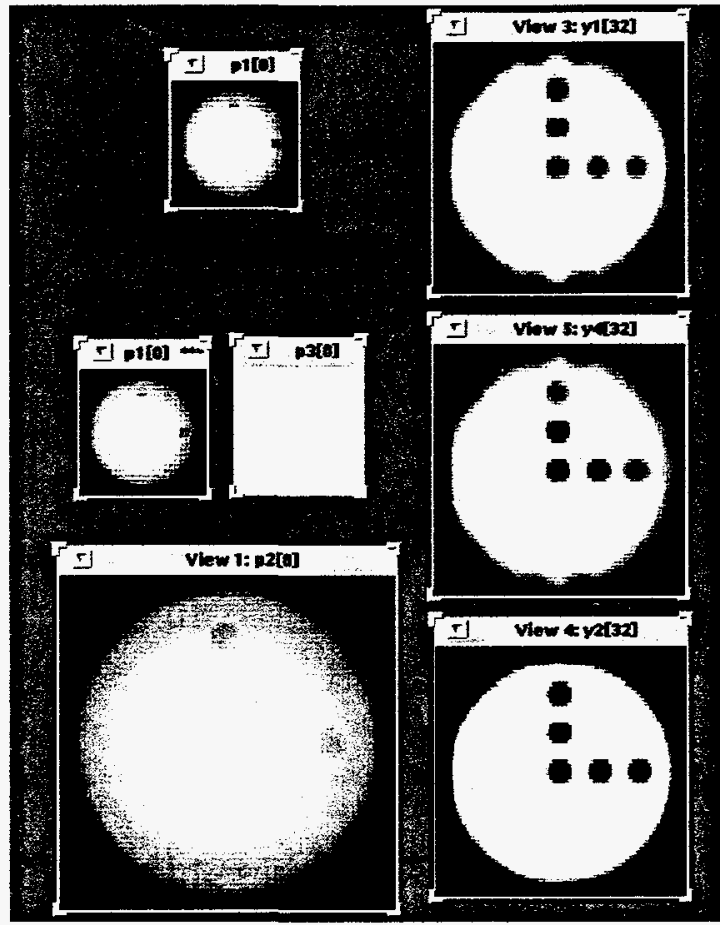

Figure 1. Simulated Radon-ROI results. Cone-beam projections (left) and vertical slices (right) of sphere with voids. Reconstructions are using LR (top), HR (bottom), and LR/ROI data with Radon-ROI (middle).

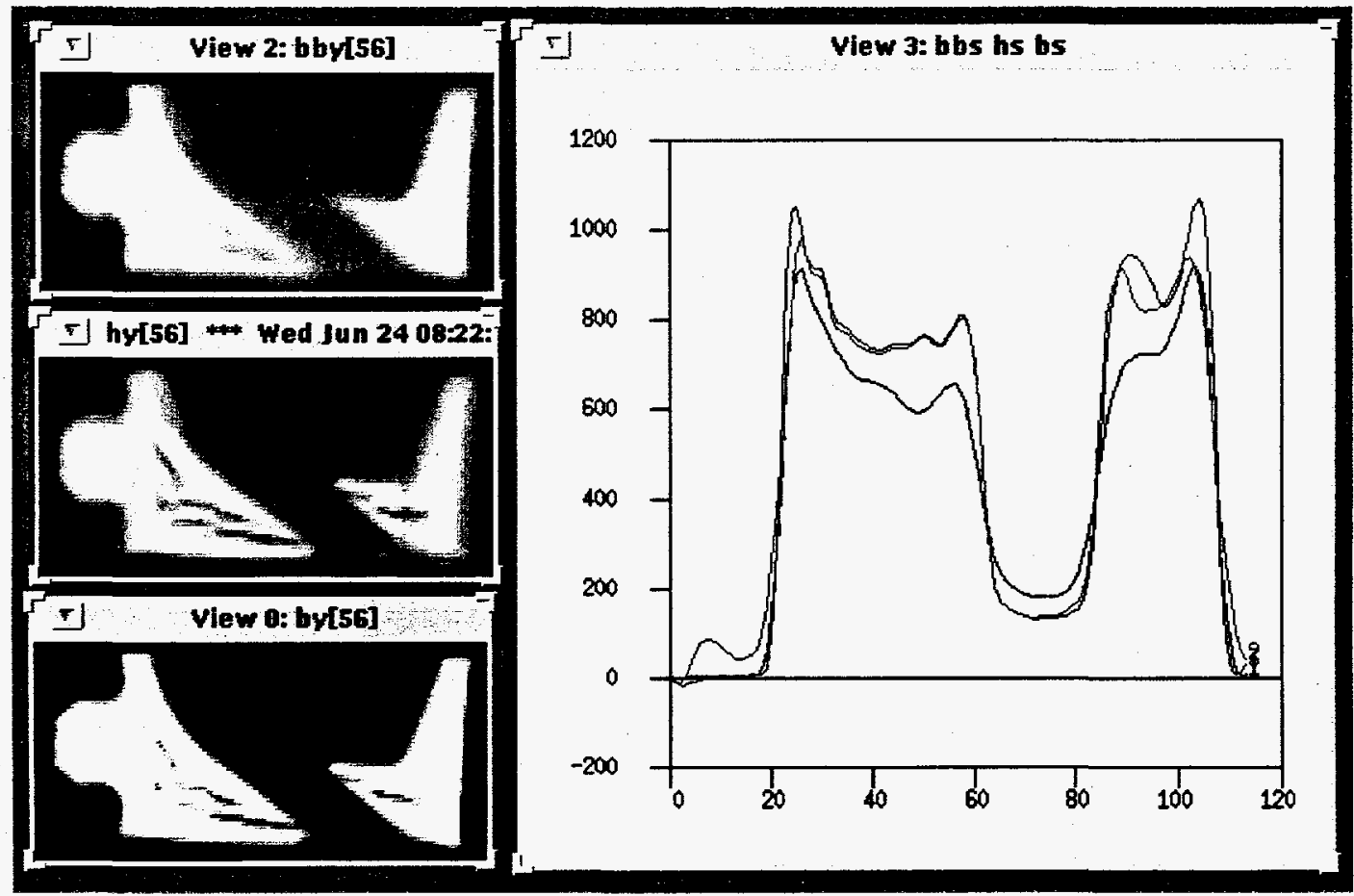

Figure 2. Experimental Radon-ROI results. Low resolution/magnification (top) and high resolution/magnification (bottom). LR/HR reconstruction with Radon-ROI (middle). 